\title{
Measurement of plasma norepinephrine and 3,4-dihydroxyphenylglycol: method development for a translational research study
}

\author{
Quin E. Denfeld ${ }^{1,2,4^{*}}$ (D), Beth A. Habecker ${ }^{1,2}$ and William R. Woodward ${ }^{2,3}$
}

\begin{abstract}
Objective: Norepinephrine (NE), a sympathetic neurotransmitter, is often measured in plasma as an index of sympathetic activity. To better understand NE dynamics, it is important to measure its principal metabolite, 3,4-dihydroxyphenylglycol (DHPG), concurrently. Our aim was to present a method, developed in the course of a translational research study, to measure NE and DHPG in human plasma using high performance liquid chromatography with electrochemical detection (HPLC-ED).

Results: After pre-purifying plasma samples by alumina extraction, we used HPLC-ED to separate and quantify NE and DHPG. In order to remove uric acid, which co-eluted with DHPG, a sodium bicarbonate wash was added to the alumina extraction procedure, and we oxidized the column eluates followed by reduction because catechols are reversibly oxidized whereas uric acid is irreversibly oxidized. Average recoveries of plasma NE and DHPG were $35.3 \pm 1.0 \%$ and $16.3 \pm 1.1 \%$, respectively, and there was no detectable uric acid. Our estimated detection limits for $\mathrm{NE}$ and DHPG were approximately $85 \mathrm{pg} / \mathrm{mL}(0.5 \mathrm{pmol} / \mathrm{mL})$ and $165 \mathrm{pg} / \mathrm{mL}(0.9 \mathrm{pmol} / \mathrm{mL})$, respectively. The measurement of NE and DHPG in human plasma has wide applicability; thus, we describe a method to quantify plasma NE and DHPG in a laboratory setting as a useful tool for translational and clinical research.
\end{abstract}

Keywords: Sympathetic nervous system, Norepinephrine, 3,4-dihydroxyphenylglycol, High performance liquid chromatography, Electrochemical detection, Human plasma

\section{Introduction}

As the principal sympathetic neurotransmitter, norepinephrine (NE), plays a critical role in regulating physiological processes $[1,2]$ and is commonly used as an index of sympathetic activity in healthy and diseased states [3-5]. In some conditions (e.g. heart failure) there is a noted increase in plasma NE [6] as a result of increases in sympathetic activity and subsequent NE "spillover" from synapses into the plasma $[7,8]$ as well as reduced reuptake of NE [9]. Elevated plasma NE portends worse outcomes such as worsening left ventricular function [10] and mortality $[7,11]$. There is wide variation, however, in

\footnotetext{
*Correspondence: denfeldq@ohsu.edu

${ }^{4}$ Present Address: School of Nursing, Oregon Health \& Science University, 3455 S.W. U.S. Veterans Hospital Road, Mail code: SN-ORD, Portland, OR 97239-2941, USA

Full list of author information is available at the end of the article
}

approaches to measure plasma NE [12-16]. Two common approaches involve either radioenzymatic [13] or enzyme immunoassay methods [14]. While these methods are well validated, they either require significant experimental considerations (e.g. use and disposal of radioisotopes) or they are limited to the measurement of $\mathrm{NE}$ alone. To gain a better sense of NE dynamics, measuring its principal metabolite, 3,4-dihydroxyphenylglycol (DHPG), provides insight into NE dynamics, offering an index of NE turnover [17-19]. As such, the measurement of both plasma NE and DHPG provides unique and complementary information about sympathetic activity in conjunction with other metrics. Our aim was to present a method, developed in the course of a translational research study, to measure NE and DHPG in human plasma using high performance liquid chromatography with electrochemical detection (HPLC-ED). This method has the benefit of a simple sample preparation process 
and concurrent measurement of both NE and DHPG, as well as being high-throughput.

\section{Main text}

\section{Materials and methods}

\section{Plasma sample and standard preparation}

We processed samples that were previously collected from heart failure patients as part of a National Institutes of Health-funded study [20]. Whole blood was centrifuged at $1000 \times g$ for $10 \mathrm{~min}$ at $5{ }^{\circ} \mathrm{C}$ to extract the plasma; the plasma was then de-identified as part of a biorepository and frozen at $-80{ }^{\circ} \mathrm{C}$. When ready to process, frozen plasma samples were thawed and then centrifuged at $8000 \times g$ for $3 \mathrm{~min}$ at $4{ }^{\circ} \mathrm{C}$ to remove insoluble material. Plasma samples (volumes ranging from 200 to $500 \mu \mathrm{L}$, depending on availability) or standards ( $500 \mu \mathrm{L}$; containing $0.1 \mu \mathrm{M}$ NE and DHPG in distilled, deionized water $\left.\left(\mathrm{ddH}_{2} \mathrm{O}\right)\right)$ were mixed with $250 \mu \mathrm{L}$ of $0.2 \mathrm{M}$ perchloric acid (PCA) containing $0.2 \mu \mathrm{M}$ dihydroxybenzylamine (DHBA; internal standard described below) and $d_{d d} \mathrm{H}_{2} \mathrm{O}$, if necessary, to make the final volume $1 \mathrm{~mL}$. An aliquot of the supernatant $(700 \mu \mathrm{L})$ was combined with $300 \mu \mathrm{L}$ of 3.0 M Tris, $\mathrm{pH} 8.5$ containing $0.1 \mathrm{mM}$ EDTA and $15 \mathrm{mg}$ of alumina (Activity Grade Super I; ICN Biomedicals). The samples were tumbled for $15 \mathrm{~min}$. The supernatant was aspirated from the alumina, and the alumina was washed once with $1 \mathrm{~mL}$ of $0.2 \mathrm{M}$ sodium bicarbonate and twice with $1 \mathrm{~mL}$ of $\mathrm{ddH}_{2} \mathrm{O}$ (with a vortex-mix and $10 \mathrm{~s}$ centrifugation between washes). Following the final water wash, $0.1 \mathrm{M} \mathrm{PCA}(150 \mu \mathrm{L})$ was added to the alumina to desorb the catechols. A $50 \mu \mathrm{L}$ aliquot of the sample was injected onto the HPLC column for analysis.

\section{High performance liquid chromatography}

The catechols were separated by reversed-phase chromatography on C18 column (Agilent Microsorb, $150 \times 4.6 \mathrm{~mm}, 5 \mu \mathrm{m}$ ) using a filtered and degassed mobile phase consisting of $75 \mathrm{mM}$ sodium phosphate ( $\mathrm{pH} 3.0$ ), $1.7 \mathrm{mM}$ sodium octane sulfonate, $1.5 \%$ acetonitrile with a flow rate of $1.0 \mathrm{~mL} / \mathrm{min}$. The mobile phase was maintained with a Shimadzu L10AD pump, and a Shimadzu SIL-20AC HT autosampler was used to inject $50 \mu \mathrm{L}$ aliquots of either sample or standard.

\section{Electrochemical detection}

An electrochemical detector (ESA Coulochem III; Bedford, MA) was used to detect and quantify the catechols. When using the oxidation protocol (detector set at $+180 \mathrm{mV}$ ) in test plasma runs, there was a large peak that co-eluted with DHPG that we identified as uric acid. Even though uric acid is poorly adsorbed onto alumina, the high amounts of uric acid that are present in the plasma of heart failure patients [21,22] result in peaks that are much larger than those for DHPG. We took advantage of the reversible oxidation of catechols but irreversible oxidation of uric acid under our conditions to analyze catechols in the plasma samples. Thus, we used an oxidation-reduction protocol in which a conditioning electrode was set at $+300 \mathrm{mV}$ to oxidize all analytes in the eluate, the first analytical electrode was set at $+150 \mathrm{mV}$ to insure complete oxidation of all analytes, and the second analytical electrode set at $-350 \mathrm{mV}$ to reduce the analytes [23]. The gain on the reducing detector was set at $50 \mathrm{nA}$ full-scale. LabSolutions (Shimadzu) software was used to collect and analyze the data (see Fig. 1 for flow diagram).

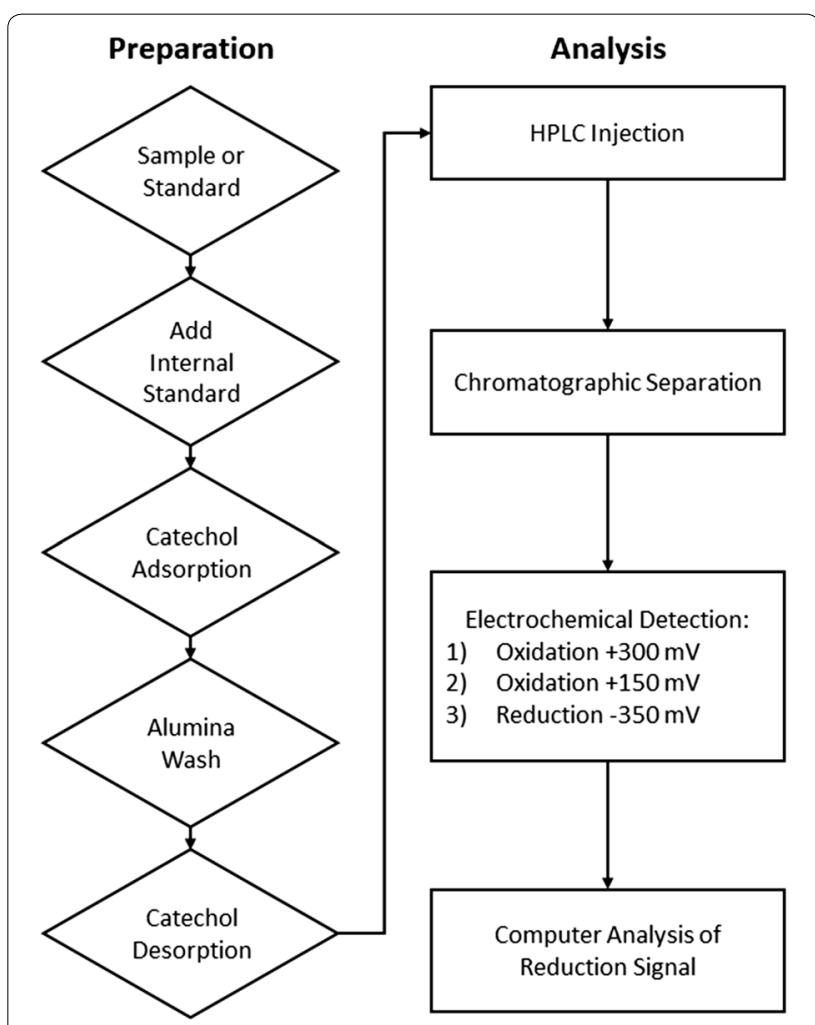

Fig. 1 Flow diagram for the oxidation-reduction protocol measuring NE and DHPG in plasma. First, to each of the samples or standards, an internal standard (i.e. DHBA) is added. Then, the catechols (NE, DHPG, and DHBA) are adsorbed onto alumina. The alumina is washed first with sodium bicarbonate and then twice with water to remove any substances not bound to the alumina. The catechols are desorbed from the alumina using 0.1 M PCA. An aliquot of the supernatant is injected onto the HPLC, and the catechols are separated by liquid chromatography on the HPLC. The catechols are first oxidized at +300 and $+150 \mathrm{mV}$ and then reduced at $-350 \mathrm{mV}$. The output of the reduction signal is analyzed by the computer software. DHBA dihydroxybenzylamine, DHPG 3,4-dihydroxyphenylglycol, HPLC high performance liquid chromatography, NE norepinephrine, $P C A$ perchloric acid 


\section{Calculation of results}

The internal standard method is commonly acknowledged to be the gold standard in quantifying analytes in complex mixtures such as plasma [24]. Since the internal standard, DHBA, behaves similarly to the plasma catechols in the alumina extraction and electrochemical detection process (but is not found in biological samples), it is possible to quantify plasma catechols in samples by referring them to standards, which both contain a standard amount of DHBA. Using the ratio of the plasma catechol peak area (i.e. NE or DHPG) to the DHBA peak area, divided by a similar ratio for the catechol standard, this ratio of ratios is then adjusted for the fraction of the sample used in the assay [correction factor (CF)] by the following equation to obtain the catechol concentration per $\mathrm{mL}$ of plasma.

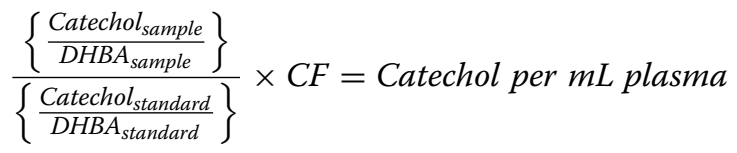

\section{Statistical analysis}

Data were analyzed using means and standard deviations. We used linear regression analysis to establish the linearity and variability of the method. All analyses were performed using GraphPad Prism version 7.02.

\section{Results}

To quantify linearity of the method, we analyzed a range of concentrations of NE $(0-0.12 \mu \mathrm{M})$, DHPG $(0-0.18 \mu \mathrm{M})$, and DHBA $(0-0.12 \mu \mathrm{M})$ that reflected the expected range of these compounds in plasma using alumina extraction and an oxidation-reduction protocol. The peak areas of NE and DHPG relative to DHBA were compared (i.e. NE:DHBA and DHPG:DHBA, respectively). The method was linear for $\mathrm{NE}\left(\mathrm{r}^{2}=0.997\right)$ and DHPG $\left(r^{2}=0.983\right)$ over this range of concentrations (Additional file 1: Figure S1).

To quantify recovery amounts, we processed known concentrations of a standard mix (containing $0.5 \mu \mathrm{M}$ DHPG, $0.5 \mu \mathrm{M}$ NE, and $0.5 \mu \mathrm{M}$ DHBA) and uric acid alone $(50 \mu \mathrm{M})$. Using both oxidation and oxidationreduction protocols, we processed the standard mix $(n=6)$ and uric acid $(n=6)$ with and without alumina extraction. Average recoveries are reported in Table 1. Despite removing $>99 \%$ of uric acid with alumina extraction and an oxidation protocol, the remaining uric acid still yielded a peak (recovery $\sim 0.075 \mu \mathrm{M}$ ) that overlaid the DHPG peak (recovery $\sim 0.155 \mu \mathrm{M}$ ). Alumina extraction coupled with the oxidation-reduction
Table 1 Recoveries following alumina extraction $(n=6)$

\begin{tabular}{lll}
\hline & \multicolumn{1}{c}{$\pm \mathbf{S D}$} & \\
\cline { 2 - 3 } & Oxidation $^{\mathbf{a}}$ & $\begin{array}{l}\text { Oxidation } \\
\text { followed } \\
\text { by reduction }\end{array}$ \\
\hline DHPG & $31.0 \pm 0.8 \%$ & $16.3 \pm 1.1 \%$ \\
Uric acid & $0.15 \pm 0.01 \%$ & $\mathrm{ND}$ \\
Norepinephrine & $49.3 \pm 1.2 \%$ & $35.3 \pm 1.0 \%$ \\
DHBA & $45.4 \pm 1.1 \%$ & $32.1 \pm 0.8 \%$
\end{tabular}

Comparison of recoveries of processed samples using the oxidation and oxidation-reduction protocols. Standard mixes $(n=6$; each containing $0.5 \mu \mathrm{M}$ DHPG, $0.5 \mu \mathrm{M} \mathrm{NE}$, and $0.5 \mu \mathrm{M} \mathrm{DHBA}$ ) and uric acid ( $n=6$; each containing $50 \mu \mathrm{M}$ ) were processed and subjected to either the oxidation protocol or the oxidationreduction protocol. We calculated recoveries of each compound based on the starting amount without alumina extraction. Even though uric acid was poorly adsorbed onto alumina, the concentration of uric acid in plasma is high compared with the catechols and even a small percentage retained obscures the DHPG peak. DHBA dihydroxybenzylamine, DHPG, 3,4-dihydroxyphenylglycol, $M$ mean, $N D$ non-detectable, $S D$ standard deviation

${ }^{a}$ Recoveries are expressed as a percentage of the starting amount

protocol eliminated the uric acid while maintaining adequate recoveries of NE and DHPG.

Representative chromatograms from plasma samples are shown in Fig. 2. In Fig. 2a, using oxidation alone, there was a large uric acid peak that co-eluted with DHPG. By including a sodium bicarbonate wash of the alumina and an electrochemical protocol involving oxidation followed by reduction, the co-eluting peak of uric acid was eliminated (Fig. 2b), unmasking the underlying DHPG peak. The peaks are negative due to the absorption of electrons by the compounds as a result of reduction. The average intra-assay coefficient of variation was $5.3 \%(n=35-40)$, and the inter-assay coefficient of variation was $4.6 \%$ $(n=4)$. Based on a plasma sample of $500 \mu \mathrm{L}$, our estimated detection limit was approximately $85 \mathrm{pg} / \mathrm{mL}$ $(0.5 \mathrm{pmol} / \mathrm{mL})$ for $\mathrm{NE}$ and $165 \mathrm{pg} / \mathrm{mL}(0.9 \mathrm{pmol} / \mathrm{mL})$ for DHPG, using a signal/noise ratio of $\geq 3$.

\section{Discussion}

Building on previous methods [23, 25], we describe a method to measure plasma NE and its principal metabolite, DHPG, that was developed as part of a translational research study. Our main findings are: (1) alumina extraction coupled with an electrochemical detection protocol involving oxidation followed by reduction eliminated a uric acid contaminant that co-eluted with DHPG, and (2) we were able to quantify NE and DHPG in plasma samples with reasonable detection limits.

An advantage of this method is the measurement of the direct principal metabolite, DHPG, which yields important information above and beyond the measurement of NE. DHPG is closer to NE in the metabolic scheme than the end metabolite, vanillylmandelic acid [18] and 


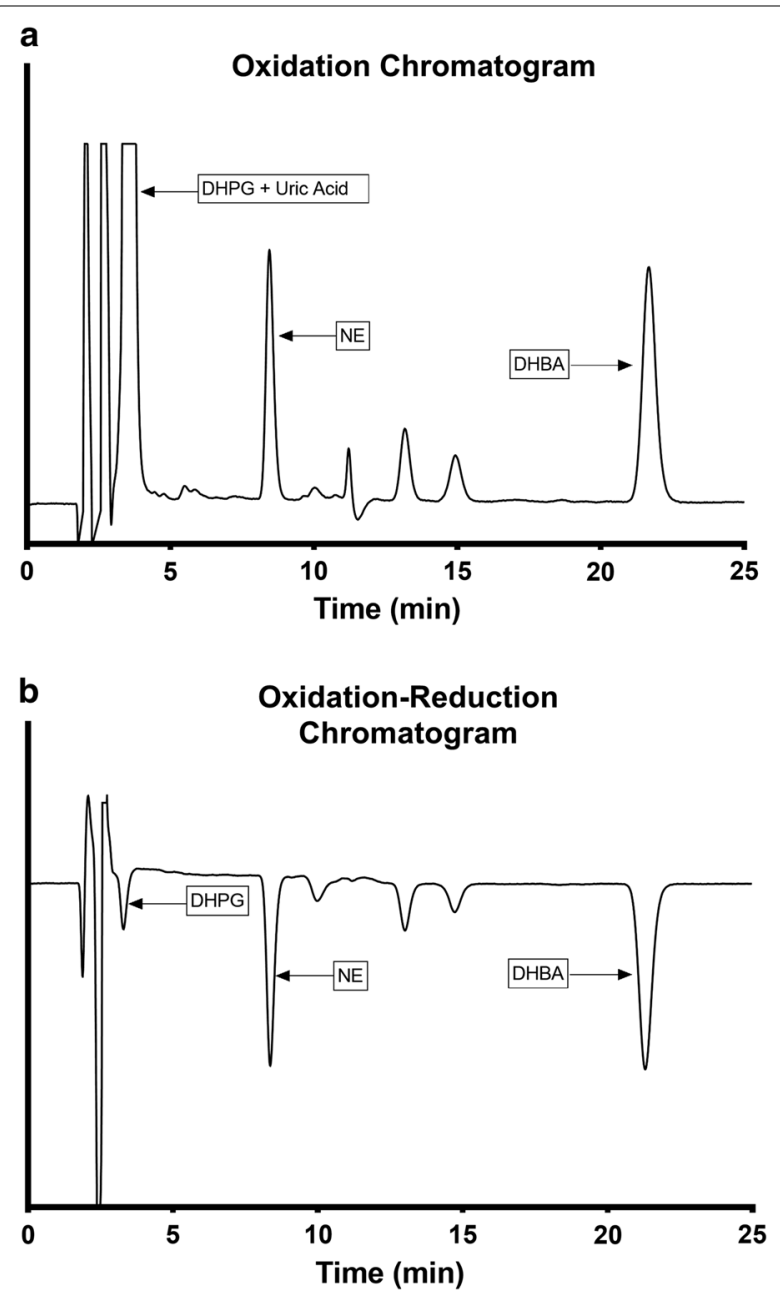

Fig. 2 Representative chromatograms of plasma samples using the oxidation protocol (a) or oxidation-reduction protocol (b). Each plasma sample was pre-purified with alumina extraction and processed with high performance liquid chromatography. Plasma samples were then either oxidized using detector settings of $+180 \mathrm{mV}$ (a) or oxidized and then reduced using detector settings of +300 , + 150, and $-350 \mathrm{mV}$ (b). DHBA dihydroxybenzylamine, DHPG 3,4-dihydroxyphenylglycol, $N E$ norepinephrine

reflects the neuronal metabolism of NE compared with the non-neuronal metabolite, normetanephrine [1]. Importantly, DHPG can be measured in the same assay as NE. Our findings demonstrate that a simple set-up with HPLC-ED, including an alumina extraction coupled with a sodium bicarbonate wash and an oxidation-reduction protocol, may rapidly increase the feasibility for clinical laboratories to detect DHPG in addition to NE.

Further consideration relates to the quantity of human plasma required for NE and DHPG detection.
Human plasma is often of limited quantity and must be carefully allocated for various assays and experiments. Complicating this circumstance, catecholamines exist in small quantities in biological fluids, which demands that bioanalytical methods must be specific and sensitive enough to detect these small quantities. In our application, we were able to detect and easily quantify amounts of NE and DHPG in plasma samples as low as $200 \mu \mathrm{L}$.

Our intent with this paper was to demonstrate how to measure plasma NE and DHPG with applicability to both translational and clinical research studies. While there are noted benefits and drawbacks to the various methods to measure plasma $\mathrm{NE}[14,15,24$, 26], researchers frequently do not have access to extensive set-ups in laboratories nor the expertise to perform complicated assays. Moreover, plasma may be the only biological fluid available, as opposed to multiplehour urine collection [27], for example. This described method has the advantage of being simple and can be set-up in any laboratory that has HPLC-ED. We outline the necessary parameters, including the sample preparation process and the chromatographic and detector settings, which permit concentration, separation, and quantification of the compounds of interest. Finally, this method was high-throughput; we were able to process about 40 samples in 2 days. In conclusion, there are multiple applications within translational and clinical research for HPLC-ED measurement of NE and its principal metabolite, DHPG, yielding clinically significant information on sympathetic activity and contributing to translational knowledge regarding key physiological processes in both health and disease.

\section{Limitations}

There are few noted limitations to this method, including the acknowledgment of well-documented analytical challenges [1, 24, 28]. First, even though we were able to estimate the neuronal reuptake of NE with DHPG levels, this method does not permit the estimation of the non-neuronal clearance of plasma NE nor the kinetics of NE. Second, we used samples collected from the forearm, which does not necessarily reflect sympathetic activity in the rest of the body because sympathetic outflow varies among tissues and organs [1]. Finally, the recovery of DHPG in the oxidation-reduction protocol was lower than NE possibly due to the sodium bicarbonate wash or less efficient reduction of DHPG. Future work to improve this method should involve developing techniques to increase the recovery of DHPG, 
particularly when the absolute concentration is necessary. Additionally, these methods should be compared with other known methods using the same plasma samples to quantify differences in reported concentrations.

\section{Additional file}

Additional file 1: Figure S1. Linearity of the response for NE (A) and DHPG (B) based on a range of physiological concentrations compared with the ratio of NE or DHPG to the internal standard, DHBA. DHBA dihydroxybenzylamine, DHPG 3,4-dihydroxyphenylglycol, NE norepinephrine.

\section{Abbreviations}

CF: correction factor; $\mathrm{ddH}_{2} \mathrm{O}$ : distilled, deionized water; DHBA: dihydroxybenzylamine; DHPG: 3,4-dihydroxyphenylglycol; HPLC-ED: high performance liquid chromatography with electrochemical detection; NE: norepinephrine; PCA: perchloric acid.

\section{Authors' contributions}

QED and BAH conceived of the initial design of the translational research study. QED and WRW developed and tested the methods, collected the data, and analyzed the results. BAH and WRW served as experts on neurochemistry and HPLC-ED methods. QED took the lead in drafting the manuscript, and BAH and WRW critically edited the manuscript. All authors read and approved the final manuscript.

\section{Author details}

${ }^{1}$ Knight Cardiovascular Institute, Oregon Health \& Science University, Portland, OR, USA. ${ }^{2}$ Department of Physiology \& Pharmacology, Oregon Health \& Science University, Portland, OR, USA. ${ }^{3}$ Department of Neurology, Oregon Health \& Science University, Portland, OR, USA. ${ }^{4}$ Present Address: School of Nursing, Oregon Health \& Science University, 3455 S.W. U.S. Veterans Hospital Road, Mail code: SN-ORD, Portland, OR 97239-2941, USA.

\section{Acknowledgements}

Not applicable.

\section{Competing interests}

The authors declare that they have no competing interests.

\section{Availability of data and materials}

Data related to the linearity, recovery, and coefficient of variation calculations are available from the corresponding author upon request.

\section{Consent for publication}

Not applicable.

\section{Ethics approval and consent to participate}

The parent study was approved by the Institutional Review Board at Oregon Health \& Science University (IRB\#7907), and written informed consent was obtained from all participants with the option to store their data and plasma samples in a biorepository. This results reported in this paper were part of an ancillary study that used a subset of de-identified plasma samples from the biorepository (from the group of participants who provided written informed consent to have their data and samples stored); this ancillary study was exempted by the Institutional Review Board at Oregon Health \& Science University as non-human subjects research (IRB\#16473).

\section{Funding}

The work reported in this paper was supported by the National Institutes of Health/National Heart, Lung, and Blood Institute (NIH/NHLBI) through a post-doctoral fellowship (for Dr. Denfeld) at Oregon Health \& Science University Knight Cardiovascular Institute (T32HL094294). Dr. Denfeld is currently supported as a Scholar of the Oregon Building Interdisciplinary Research Careers in Women's Health K12 Program funded by the Eunice Kennedy Shriver National Institute of Child Health \& Human Development of the $\mathrm{NIH}$ under Award Number K12HD043488. Plasma samples were collected as part of a parent study that was funded by the National Institutes of Health/ National Institute of Nursing Research (R01NR013492; Lee) and supported by the National Center for Advancing Translational Sciences of the National Institutes of Health (UL1TR000128). Drs. Habecker and Woodward are currently funded by the NIH/NHLBI (R01HL093056; Habecker). The content is solely the responsibility of the authors and does not necessarily represent the official views of the $\mathrm{NIH}$.

\section{Publisher's Note}

Springer Nature remains neutral with regard to jurisdictional claims in published maps and institutional affiliations.

Received: 5 February 2018 Accepted: 11 April 2018

Published online: 19 April 2018

\section{References}

1. Goldstein DS, Eisenhofer G, Kopin IJ. Sources and significance of plasma levels of catechols and their metabolites in humans. J Pharmacol Exp Ther. 2003;305:800-11.

2. Goldstein DS. Catecholamines 101. Clin Auton Res. 2010:20:331-52.

3. Florea VG, Cohn JN. The autonomic nervous system and heart failure. Circ Res. 2014;114:1815-26.

4. Esler M, Jennings G, Lambert G, Meredith I, Horne M, Eisenhofer G. Overflow of catecholamine neurotransmitters to the circulation: source, fate, and functions. Physiol Rev. 1990;70:963-85.

5. Rasch B, Dodt C, Mölle M, Born J. Sleep-stage-specific regulation of plasma catecholamine concentration. Psychoneuroendocrinology. 2007;32:884-91.

6. Chidsey CA, Harrison DC, Braunwald E. Augmentation of the plasma nor-epinephrine response to exercise in patients with congestive heart failure. N Engl J Med. 1962;267:650-4.

7. Kaye DM, Lefkovits J, Jennings GL, Bergin P, Broughton A, Esler MD. Adverse consequences of high sympathetic nervous activity in the failing human heart. J Am Coll Cardiol. 1995;26:1257-63.

8. Hasking GJ, Esler MD, Jennings GL, Burton D, Johns JA, Korner PI. Norepinephrine spillover to plasma in patients with congestive heart failure: evidence of increased overall and cardiorenal sympathetic nervous activity. Circulation. 1986;73:615-21.

9. Eisenhofer G, Friberg P, Rundqvist B, Quyyumi AA, Lambert G, Kaye DM, Kopin IJ, Goldstein DS, Esler MD. Cardiac sympathetic nerve function in congestive heart failure. Circulation. 1996;93:1667-76.

10. Thomas JA, Marks BH. Plasma norepinephrine in congestive heart failure. Am J Cardiol. 1978;41:233-43.

11. Rector TS, Olivari MT, Barry Levine T, Francis GS, Cohn JN. Predicting survival for an individual with congestive heart failure using the plasma norepinephrine concentration. Am Heart J. 1987;114:148-52.

12. Evans MI, Halter JB, Porte D Jr. Comparison of double- and single-isotope enzymatic derivative methods for measuring catecholamines in human plasma. Clin Chem. 1978:24:567-70.

13. Henry DP, Starman BJ, Johnson DG, Williams RH. A sensitive radioenzymatic assay for norepinephrine in tissues and plasma. Life Sci. 1975;16:375-84.

14. Westermann J, Hubl W, Kaiser N, Salewski L. Simple, rapid and sensitive determination of epinephrine and norepinephrine in urine and plasma by non-competitive enzyme immunoassay, compared with HPLC method. Clin Lab. 2002;48:61-71.

15. Raum WJ. Methods of plasma catecholamine measurement including radioimmunoassay. Am J Physiol. 1984;247:E4-12.

16. Hjemdahl P. Catecholamine measurements by high-performance liquid chromatography. Am J Physiol. 1984;247:E13-20.

17. Goldstein DS, Eisenhofer G, Stull R, Folio CJ, Keiser HR, Kopin IJ. Plasma dihydroxyphenylglycol and the intraneuronal disposition of norepinephrine in humans. J Clin Invest. 1988;81:213-20.

18. Peaston RT, Weinkove C. Measurement of catecholamines and their metabolites. Ann Clin Biochem. 2004;41:17-38.

19. Izzo JL Jr, Thompson DA, Horwitz D. Plasma dihydroxyphenylglycol (DHPG) in the in vivo assessment of human neuronal norepinephrine metabolism. Life Sci. 1985:37:1033-8. 
20. Lee CS, Mudd JO, Gelow JM, Nguyen T, Hiatt SO, Green JK, Denfeld QE, Bidwell JT, Grady KL. Background and design of the profiling biobehavioral responses to mechanical support in advanced heart failure study. J Cardiovasc Nurs. 2014;29:405-15.

21. Anker SD, Doehner W, Rauchhaus M, Sharma R, Francis D, Knosalla C, Davos CH, Cicoira M, Shamim W, Kemp M, et al. Uric acid and survival in chronic heart failure: validation and application in metabolic, functional, and hemodynamic staging. Circulation. 2003;107:1991-7.

22. Kaufman M, Guglin M. Uric acid in heart failure: a biomarker or therapeutic target? Heart Fail Rev. 2013;18:177-86.

23. Eisenhofer G, Goldstein DS, Stull R. Simultaneous liquid-chromatographic determination of 3,4-dihydroxyphenylglycol, catecholamines, and 3,4-dihydroxyphenylalanine in plasma, and their responses to inhibition of monoamine oxidase. Clin Chem. 1986;32:3030-3.

24. Hjemdahl P. Plasma catecholamines-analytical challenges and physiological limitations. Baillieres Best Pract Res Clin Endocrinol Metab. 1993;7:307-53.
25. Musso NR, Vergassola C, Pende A, Lotti G. Reversed-phase HPLC separation of plasma norepinephrine, epinephrine, and dopamine, with threeelectrode coulometric detection. Clin Chem. 1989;35:1975-7.

26. Bicker J, Fortuna A, Alves G, Falcão A. Liquid chromatographic methods for the quantification of catecholamines and their metabolites in several biological samples_a review. Anal Chim Acta. 2013;768:12-34.

27. White IR, Brunner EJ, Barron JL. A comparison of overnight and 24 hour collection to measure urinary catecholamines. J Clin Epidemiol. 1995:48:263-7.

28. Esler M, Kaye D. Measurement of sympathetic nervous system activity in heart failure: the role of norepinephrine kinetics. Heart Fail Rev. 2000;5:17-25.
Ready to submit your research? Choose BMC and benefit from:

- fast, convenient online submission

- thorough peer review by experienced researchers in your field

- rapid publication on acceptance

- support for research data, including large and complex data types

- gold Open Access which fosters wider collaboration and increased citations

- maximum visibility for your research: over $100 \mathrm{M}$ website views per year

At BMC, research is always in progress.

Learn more biomedcentral.com/submissions 